\title{
Melosh rotation: source of the proton's missing spin
}

\author{
Bo-Qiang Ma \\ Center of Theoretical Physics, CCAST (World Laboratory), Beijing, People's \\ Republic of China \\ and \\ Institute of High Energy Physics, Academia Sinica, PO Box 918 (4), Beijing 100039, \\ People's Republic of China
}

\begin{abstract}
It is shown that the observed small value of the integrated spin structure function for protons could be naturally understood within the naive quark model by considering the effect from Melosh rotation. The key to this problem lies in the fact that the deep inelastic process probes the light-cone quarks rather than the instantform quarks, and that the spin of the proton is the sum of the Melosh rotated light-cone spin of the individual quarks rather than simply the sum of the light-cone spin of the quarks directly.
\end{abstract}

The spin content of the proton has received extensive attention from the particle physics community recently. The reason for this is that the European Muon Collaboration (EMC) found [1] from their polarized muon proton data a much smaller value of the integrated spin structure function for protons compared with that from the Ellis-Jaffe sum rules 2. This small integrated spin structure function combined with the Bjorken sum rule [3] was interpreted as the evidence that a very small fraction of the proton's spin is provided by the spin of its quarks. This conclusion, if true, is of course startling because it is in clear contradiction with the previous theoretical expectations [4]. Hence, many papers have been devoted to this problem and many complicated models for the proton's missing spin have been proposed. In this letter, we indicate, however, that the small value of the proton's integrated spin structure function could be naturally understood in the naive quark model (ком) by considering the effect of the Melosh rotation [5, 6].

The key to this problem lies in two very simple but in practice often mistakenly treated or ignored facts. The first is that the deep inelastic lepton scattering process is a probe of the light-cone (or current) quarks rather than the instant-form (or constituent) quarks [7, 8, 9]. The second is that in light-front dynamics the spin of the proton is not simply the sum of the spin of the individual quarks but the sum of the Melosh rotated spin of the light-cone quarks [10, 11. The theoretical bases for the two facts can be traced back to the old work of Dirac's relativistic Hamiltonian dynamics [12], Weinberg's 
infinite momentum technique [13], and Wigner's spin state rotation [14]. The first fact leads naturally to the conclusion that the quark's spin measured in deep inelastic lepton scattering is the light-cone spin rather than the instant-form spin. Taken in conjunction with the second fact, we can conclude that there is no need to require that the sum of the quark's spin measured in deep inelastic process be equal to the proton's spin.

In the following, we simply present an intuitive model to evaluate the effect from Melosh rotation. We start from the conventional instant-form (T) NQM SU(6) proton wavefunction

$$
\left|p_{T}^{\uparrow}\right\rangle=\left(2 u_{T}^{\uparrow} u_{T}^{\uparrow} d_{T}^{\downarrow}-u_{T}^{\uparrow} u_{T}^{\downarrow} d_{T}^{\uparrow}-u_{T}^{\downarrow} u_{T}^{\uparrow} d_{T}^{\uparrow}\right) / \sqrt{6} \text { (+cyclic permutation) }
$$

one finds $\triangle u_{T}=\frac{4}{3}, \triangle d_{T}=-\frac{1}{3}$ and $\triangle s_{T}=0$. If the deep inelastic process is a probe of the instant-form quarks, we expect, respectively, the integrated spin structure function for protons

$$
\int \mathrm{g}_{1}^{p} d x=\frac{1}{2}\left(\frac{4}{9} \triangle u+\frac{1}{9} \triangle d\right)=\frac{5}{18}=0.278
$$

and that for neutrons

$$
\int \mathrm{g}_{1}^{n} d x=\frac{1}{2}\left(\frac{1}{9} \triangle u+\frac{4}{9} \triangle d\right)=0
$$

together with the proton's spin sum rule

$$
\left(\triangle S_{Z}^{T}\right)_{u+d+s}=\frac{1}{2}\left(\triangle u_{T}+\triangle d_{T}\right)=\frac{1}{2}
$$

which means that the proton's full spin is carried by its valence quarks.

The instant-form $(\mathrm{T})$ quark states $q_{T}^{s}$ and the front-form $(\mathrm{F})$ quark states $q_{F}^{s}$ are related by the Melosh rotation [5, 6, 10, 11]

$$
q_{F}^{s}=\sum_{s^{\prime}} M_{s^{\prime} s}(R) q_{T}^{s^{\prime}}
$$

with the Melosh rotation operator defined by

$$
R=\left(m+k_{0}+k_{3}+i \varepsilon_{i j 3} \sigma_{i} k_{j}\right) /\left[2\left(k_{0}+k_{3}\right)\left(m+k_{0}\right)\right]^{1 / 2}
$$

in specifying $q_{F}^{s}$ and $q_{T}^{s}$ by the two-component Pauli spinors. From (5) , we get, inversely,

$$
q_{T}^{\uparrow}=w\left[\left(k^{+}+m\right) q_{F}^{\uparrow}-k^{R} q_{F}^{\downarrow}\right] \quad q_{T}^{\downarrow}=w\left[\left(k^{+}+m\right) q_{F}^{\downarrow}+k^{L} q_{F}^{\uparrow}\right]
$$

in which $w=\left[2 k^{+}\left(m+k_{0}\right)\right]^{-1 / 2}, k^{R, L}=k_{1} \pm i k_{2}, k^{+}=k_{0}+k_{3}$ and $k_{0}=\left(m^{2}+\mathbf{k}^{2}\right)^{1 / 2}$. We see from (7) that the light-cone spin carried by an instant-form quark should be its instant-form spin multiplied by a factor

$$
M_{q}=\left[\left(k^{+}+m\right)^{2}-\mathbf{k}_{\perp}^{2}\right] /\left[2 k^{+}\left(m+k_{0}\right)\right] .
$$

Therefore we can identify

$$
\triangle q_{F}=\left\langle M_{q}\right\rangle \triangle q_{T}
$$

in which $M_{q}$ is the contribution from the Melosh rotation.

We simply assume that the quark momentum-space wavefunction of the proton is described by the harmonic oscillator wavefunction

$$
\Psi(\mathbf{k})=\pi^{-3 / 4} \alpha^{-3 / 2} \exp \left(-\mathbf{k}^{2} / 2 \alpha^{2}\right) .
$$


We know from previous work [15] that this wavefunction is good in describing the static properties of hadrons at low momentum scale in adopting the harmonic scale $\alpha \approx 330$ $\mathrm{MeV}$ and the quark mass $m \approx 330 \mathrm{MeV}$. At high momentum scale one may expect that $m$ becomes smaller or $\alpha$ becomes larger [15]. Hence the expectation value of $M_{q}$ may be evaluated by

$$
\left\langle M_{q}\right\rangle=\int d^{3} \mathbf{k} M_{q}|\Psi(\mathbf{k})|^{2} .
$$

As deep inelastic scattering is a probe of the light-cone quarks, we should use $\triangle q_{F}$ rather than $\triangle q_{T}$ in equations (2)-(3) to calculate the integrated spin structure functions for protons and neutrons. Assuming $M_{u}=M_{d}$ and adopting $m, \alpha$ to be $134 \mathrm{MeV}, 330$ $\mathrm{MeV}$ or $330 \mathrm{MeV}, 815 \mathrm{MeV}$ respectively, we obtain

$$
\int \mathrm{g}_{1}^{p} d x=\frac{1}{2}\left(\frac{4}{9} \triangle u_{F}+\frac{1}{9} \triangle d_{F}\right)=\frac{5}{18}=0.126
$$

and

$$
\int \mathrm{g}_{1}^{n} d x=\frac{1}{2}\left(\frac{1}{9} \triangle u_{F}+\frac{4}{9} \triangle d_{F}\right)=0
$$

together with

$$
\left(\triangle S_{z}^{F}\right)_{u+d}=\frac{1}{2}\left(\triangle u_{F}+\triangle d_{F}\right)=0.227
$$

which means that the sum of the light-cone spin of the valence quarks is only $45.4 \%$ of the proton's spin. From (12) we see that the above intuitive picture could naturally explain the small EMC data of the proton's integrated spin structure function with reasonable parameters.

One can easily find that the above results are quantitatively inconsistent with the Bjorken sum rule. This comes from the adoption of the NQM SU(6) proton wavefunction and the assumption $M_{u}=M_{d}$. Actually the proton's instant-form wavefunction should be

$$
\left|p_{T}\right\rangle=a_{0}|u u d\rangle_{T}+a_{1}|u u d q \bar{q}\rangle_{T}+a_{2}|u u d g\rangle_{T}+\cdots
$$

in which the high Fock state contributions could change $\triangle u_{T}$ and $\triangle d_{T}$ from the values $\frac{4}{3}$ and $-\frac{1}{3} .\left\langle M_{u}\right\rangle$ and $\left\langle M_{d}\right\rangle$ may also be different since there are two $u$ valence quarks and one $d$ valence quark in the proton. Bearing the above consideration in mind, we start from the most recent EMC data

$$
\int \mathrm{g}_{1}^{p} d x=\frac{1}{2}\left(\frac{4}{9} \triangle u_{F}+\frac{1}{9} \triangle d_{F}\right)=\frac{5}{18}=0.126
$$

and the Bjorken sum rule

$$
\int\left(\mathrm{g}_{1}^{p}-\mathrm{g}_{1}^{n}\right) d x=\frac{1}{6}\left(\triangle u_{F}-\triangle d_{F}\right)=\frac{1}{6} \mathrm{~g}_{A} / \mathrm{g}_{V}
$$

with $\mathrm{g}_{A} / \mathrm{g}_{V}=1.259$ determined from neutron $\beta$ decay [16] to evaluate the values of $\triangle u_{T}, \triangle d_{T},\left\langle M_{u}\right\rangle$ and $\left\langle M_{d}\right\rangle$. In order to simplify the discussion, we neglect the possible 
effects from the sea or gluon polarization $\$$ and from the quark or gluon orbital angular momentum. From (16) and (17), we obtain

$$
\triangle u_{F}=\left\langle M_{u}\right\rangle \triangle u_{T}=0.705 \quad \triangle d_{F}=\left\langle M_{d}\right\rangle \triangle d_{T}=-0.554
$$

and the sum of the light-cone spin of the valence quarks

$$
\left(\triangle S_{z}^{F}\right)_{u+d}=\frac{1}{2}\left(\triangle u_{F}+\triangle d_{F}\right)=0.076
$$

which is very small. We know that $\triangle u_{T, F}, \triangle d_{T, F},\left\langle M_{u}\right\rangle$ and $\left\langle M_{d}\right\rangle$ should meet the general requirements

$$
-2 \leq \triangle u_{T, F} \leq 2 \quad-1 \leq \triangle d_{T, F} \leq 1 \quad 0 \leq\left\langle M_{u, d}\right\rangle \leq 1
$$

and the spin sum rule

$$
\frac{1}{2}\left(\triangle u_{T}+\triangle d_{T}\right)=\frac{1}{2}
$$

The combination of equations (18), (20) and (21) leads to the constraints

$$
\begin{aligned}
& 1.554 \leq \triangle u_{T} \leq 2 \quad 0.352 \leq\left\langle M_{u}\right\rangle \leq 0.454 \quad-1 \leq \triangle d_{T} \leq-0.554 \\
& 0.554 \leq\left\langle M_{d}\right\rangle \leq 1 .
\end{aligned}
$$

Therefore in order to satisfy both the EMC data and the Bjorken sum rule simultaneously, it is necessary that $\left\langle M_{u}\right\rangle \neq\left\langle M_{d}\right\rangle, \triangle u_{T} \neq \frac{4}{3}$, and $\triangle d_{T} \neq-\frac{1}{3}$; i.e. the proton's instant-form valence quark distribution should be different from that of the NQm $\mathrm{SU}(6)$ wavefunction, and the $u$ quark and the $d$ quark should have different momentum-space wavefunctions in the proton. We are also interested to see that the Melosh rotation is also an important source for the depletion of $g_{A} / g_{V}$ relative to the value $\frac{5}{3}$ expected from the $\mathrm{SU}(6)$ naive quark model. This is a significant source in comparison with other sources such as the effect from the quark transverse momenta [18] and the effect due to 'small' components in the quark's Dirac spinors in the bag model [19] or in quark-confining potentials [20].

In summary, we present in this letter a very simple model in which the EMC results of the proton's integrated spin structure function could be naturally explained within the naive quark model by considering the effect from Melosh rotation. This model does not necessarily invalidate the Bjorken sum rule if we impose some constraints on the Fock state wavefunction of the proton. This work is based on two very simple hut profound facts which have sound bases both theoretically and experimentally. Though the quantitative results in this letter may be changed by the complicated effects from the sea and gluon polarizations and by contributions from the orbital angular momentum, or by the anomalous gluon contributions via the U(l) axial anomaly, the effect from Melosh rotation should be of fundamental importance in the spin content of hadrons and therefore should not be ignored. We think the effect revealed in this letter should have also manifested itself in a number of high energy processes, and therefore requires further theoretical and experimental works.

$\ddagger$ Close [17] indicated recently that the magnitude of the (strange) sea polarization is likely to be significantly nearer to zero than is being assumed in much of the current literature. 


\section{References}

[1] Ashman J et al (EMC) 1988 Phys. Lett. 206B 364; 1989 Nucl. Phys. B 3281

[2] Ellis J and Jaffe R L 1974 Phys. Rev. D 9 1444; 1974 Phys. Rev. D 101699 (E)

[3] Bjorken J D 1971 Phys. Rev. D 1 1376; 1966 Phys. Rev. 1481467

[4] Jaffe R L and Manohar A 1990 Nucl. Phys. B 337509

[5] Melosh H J 1974 Phys. Rev. D 91095

[6] Berestetskii V R and Terent'ev M V 1976 Yad. Fiz. 241044 (Engl. transl. 1976 Sol. J. Nucl. Phys. 24 547)

Kondratyuk L A and Terent'ev M V 1980 Yad. Fiz. 311087 (Engl. transl. 1980 Sol. J. Nucl. Phys. 31 561)

[7] Drell S D, Levy D J, and Yan T -M 1969 Phys. Rev. 187 2159; 1970 Phys. Rev. D 11035 Drell S D and Yan T-M 1971 Ann. Phys., NY 66578

[8] Brodsky S J 1982 Lectures on Lepton-Nucleon Scattering and Quantun Chromodynamics ed A Jaffe and D Rude (Boston: Birkhäuser) p 255

Brodsky S J, Huang T, and Lepage G P, 1983 Particles and Fields ed A Z Capri and A N Kamal (New York: Plenum) p 143

[9] Ma B -Q 1989 PhD dirssertation Peking University Ma B -Q and Sun J 1990 J.Pkys. G:Nucl. Part. Phys. 16 823; 1990 High Energy Phys. Nucl. Phys. (Chinese ed) 14 416; 1990 Int. J. Mod. Phys. A 6345

[10] Coester F 1965 Helv. Phys. Acta 38 7; 1987 Constraint's Theory and Relativisitic Dynamics ed G Longhi and L Lusanna (Singapore: World Scientific) p 159; 1986 The Three-Body Force in the Three-Nucleon System ed B L Berman and B F Gibson (New York:Springer) p 472

[11] Chung P L, Coester F, Keister B D and Polyzou W N 1988 Phys. Rev. C 372000 Keister B D 1989 Nuclear and Particle Physics on the Light Cone ed M B Johnson and L S Kisslinger (Singapre: World Scientific) p 439

[12] Dirac P A M 1949 Rev. Mod. Phys. 21392

[13] Weinberg S 1966 Phys. Rev. 1501313

[14] Wigner E 1939 Ann. Math. 40149

[15] Isgur N 1980 The New Aspects of Subnuclear Physics ed A Zichichi (NewYork: Plenum) p 107 Dziembowski Z 1988 Phys. Rev. D 37778

[16] Particle Data Group, Yost G P et al 1988 Phys. Lett. 204B 1

[17] Close F E 1990 Phys. Rev. Lett. 64361

[18] Bogoloubov P N 1968 Ann. Inst. H Poincare 8163

[19] Close F E 1979 An Introduction to Quarks and Partons (New York: Academic) p 115, 419

[20] Tegen R 1989 Phys. Rev. Lett. 621724 and references therein 